\title{
The ivy sign
}

\author{
Authors: \\ Nasreen Mahomed ${ }^{1,2}$ \\ Evance Chisama ${ }^{1}$ \\ Sanjay Prabhu ${ }^{3}$ \\ Affiliations: \\ ${ }^{1}$ Department of Radiology, \\ University of the \\ Witwatersrand, South Africa \\ ${ }^{2}$ Consultant radiologist, Chris \\ Hani Baragwanath Hospital, \\ South Africa \\ ${ }^{3}$ Department of Radiology, \\ Boston Children's Hospital, \\ Harvard Medical School, \\ United States of America

\section{Correspondence to:} \\ Nasreen Mahomed \\ Email: \\ nasreen.mahomed@wits. \\ ac.za

\section{Postal address:} \\ Private Bag X3, University \\ of the Witwatersrand, \\ Johannesburg 2050, \\ South Africa

\section{Dates:} \\ Received: 14 Apr. 2014 \\ Accepted: 17 Oct. 2014 \\ Published: 05 Dec. 2014 \\ How to cite this article: \\ Mahomed N, Chisama \\ E, Prabhu S. The ivy sign. \\ S Afr J Rad. 2014;18(1); Art. \\ \#622, 3 pages. http://dx.doi/ \\ org.10.4102/sajr.v18i1.622

\section{Copyright:} \\ (C) 2014. The Authors. \\ Licensee: AOSIS \\ OpenJournals. This work is \\ licensed under the Creative \\ Commons Attribution \\ License.
}

\section{Read online:}

The ivy sign refers to diffuse bilateral leptomeningeal enhancement on post- contrast T1-weighted magnetic resonance imaging (MRI) and increased signal intensity in bilateral subarachnoid spaces and perivascular spaces on T2-weighted fluid attenuation inversion recovery (FLAIR) MRI sequences in patients with moyamoya disease.

\section{Signs of diffuse bilateral leptomeningeal enhancement}

Moyamoya disease is an idiopathic cerebrovascular occlusive disorder characterised by progressive stenosis of the distal aspect of the internal carotid arteries, the proximal aspects of the middle cerebral arteries and the anterior cerebral arteries, with subsequent collateral vessel formation. The ivy sign represents neovascularisation over the cerebral cortex via leptomeningeal anastomosis through collateral circulation, and represents diffuse engorgement of the pia vasculature.

The ivy sign was first described in 1995, so called because of its resemblance to 'ivy creeping on stone'. The sign refers to diffuse bilateral leptomeningeal enhancement on post-contrast T1-weighted magnetic resonance imaging (MRI) (Figure 1) and increased signal intensity in bilateral subarachnoid spaces and perivascular spaces on T2-weighted fluid attenuation inversion recovery (FLAIR) MRI (Figure 2) sequences in patients with moyamoya disease. ${ }^{1,2}$

Moyamoya disease is an idiopathic cerebrovascular occlusive disorder characterised by progressive stenosis of the distal aspect of the internal carotid arteries (ICAs), the proximal aspects of the middle cerebral arteries (MCAs) and the anterior cerebral arteries (ACAs), with subsequent collateral vessel formation. Moyamoya is a Japanese term, meaning a puff or spiral of smoke, and describes the cloudlike lenticulostriate and thalamostriate collaterals on angiography. The three basic collateral pathways involved include the basal moyamoya vessels from perforators, leptomeningeal collateral vessels from the posterior cerebral artery, and the transdural collateral vessels from the external carotid artery (Figure 3).,34

The leptomeningeal enhancement on post-contrast T1-weighted MRI (Figure 1), seen in the ivy sign in patients with moyamoya disease, represents neovascularisation over the cerebral cortex via leptomeningeal anastomosis through collateral circulation. This develops from the anastomosis of external carotid collateral arteries and ICAs, and represents diffuse engorgement of the pia vasculature. ${ }^{2}$ This collateral flow is very slow owing to its retrograde direction via leptomeningeal anastomosis. From this phenomenon, the engorged pial network shows postcontrast enhancement. ${ }^{2}$ Recent literature suggests that 3.0-Tesla MR imaging is superior to 1.5-Tesla MR imaging in visualising the ivy sign and associated changes in moyamoya disease because of the higher resolution and increased signal-to-noise ratio and contrast-to-noise ratio, thus enabling better depiction of pathological small vessel segments, with greater angiographic detail. ${ }^{5}$

It has become evident in moyamoya disease that, when successful bypass surgery is performed, the leptomeningeal enhancement (the ivy sign) on contrast-enhanced T1-weighted sequences decreases or disappears. This classical finding implies that the ivy sign in moyamoya disease represents cortical insufficiency rather than reactive hyperaemia. ${ }^{6}$ In the postoperative period following pial synangiosis, the resolution of the ivy sign in conjunction with development of more robust collaterals underlying the site of pial synangiosis indicates successful revascularisation and improved, faster flow in the involved areas. ${ }^{7,8}$ The ivy sign can be seen in new areas following surgery, and this can be an indication that slow flow in new collaterals has developed underlying the synangiosis. These areas need to be carefully assessed on serial studies to ensure that the flow improves over time and is matched by flow enhancement in collaterals on time-of-flight or postcontrast MR angiography (MRA). ${ }^{7,89}$

Pitfalls of the ivy sign include the leptomeningeal enhancement on post-contrast T1-weighted MRI in cases of meningitis and meningeal carcinomatosis characterised by inflammation or 


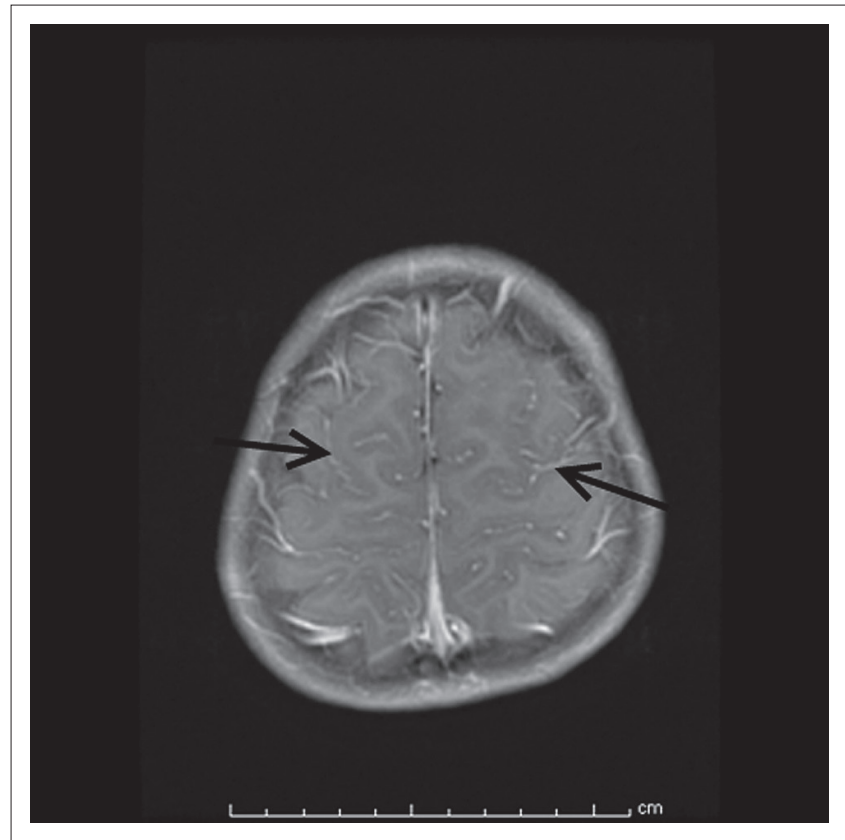

MRI, magnetic resonance imaging.

FIGURE 1: Post-contrast T1-weighted MRI demonstrating the ivy sign, namely diffuse leptomeningeal enhancement (arrows) resembling ivy creeping on stone.

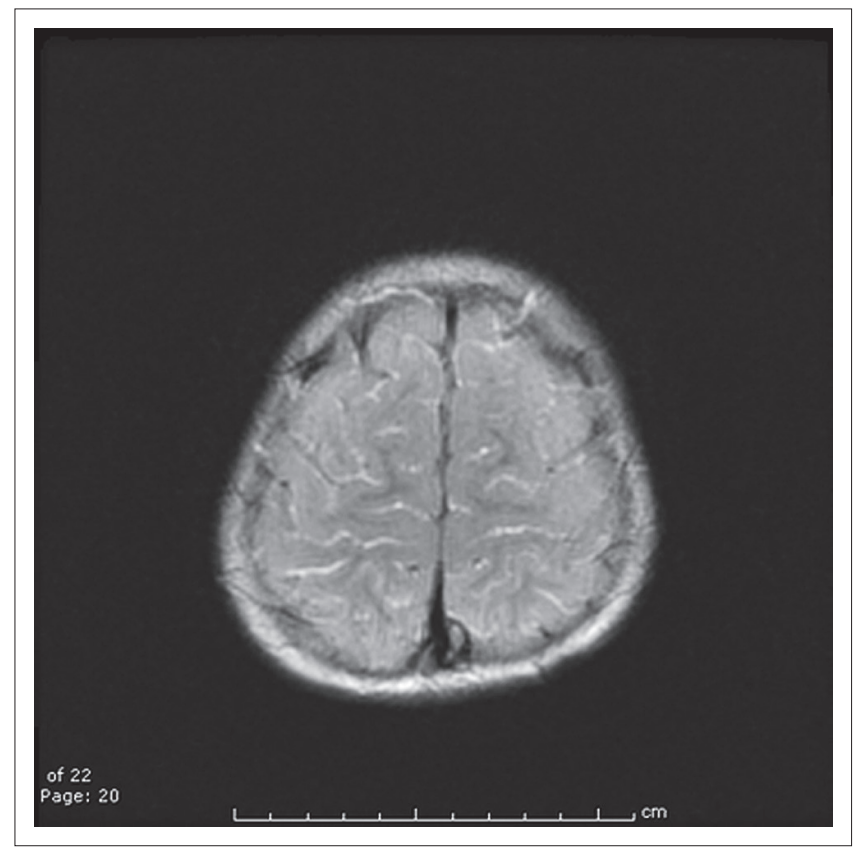

FLAIR MRI, fluid attenuation inversion recovery magnetic resonance imaging.

FIGURE 2: T2-weighted FLAIR MRI sequences demonstrating the ivy sign, namely increased signal intensity in the bilateral subarachnoid spaces and perivascular spaces. This represents neovascularisation over the cerebral cortex via leptomeningeal anastomosis through collateral circulation that develops from the diffuse pial arterial network.

carcinomatous infiltration of the leptomeninges, with or without involvement of the small leptomeningeal vessels. ${ }^{1}$

\section{Experiences at Boston Children's Hospital}

Owing toinherentdifficulties with post-contrastMRAinyoung children, including intravenous access, need for sedation and

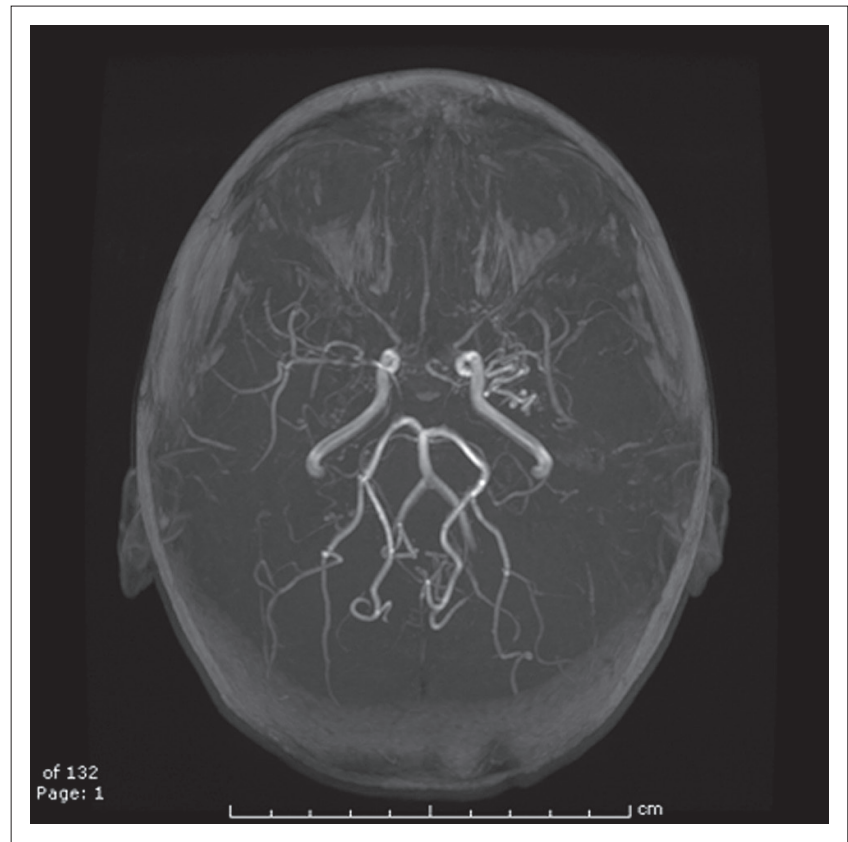

MR, magnetic resonance.

FIGURE 3: MR angiography demonstrating moyamoya disease, with stenosis of the distal aspect of the internal carotid arteries, the proximal aspects of the middle cerebral arteries and the anterior cerebral arteries, with subsequent collateral vessel formation resembling a puff or spiral of smoke, which describes the cloud-like lenticulostriate and thalamostriate collaterals.

limitations to pump injection, we rely on a combination of 3D time-of-flight MRA and arterial spin labelling studies with several time delays to assess the cerebral bloodflow in patients with moyamoya disease. With our experience of over 400 paediatric patients at Boston Children's Hospital who have undergone pial synangiosis and correlation of a large proportion of cases with conventional angiography, we found that post-contrast MRA is not necessary in most cases to help assess post-surgical collateral flow. Arterial spin labelling studies are challenging as bloodflow through the synangiosis comes in via a longer pathway (external carotid arteries and its branches), and this necessitates longer delay times to assess the label in this portion of the brain. Absence of the ivy sign in previously involved areas and new vessels on time-of-flight suffices in most cases to assess whether the surgery has worked or not. Conventional angiography is used in equivocal cases at our institution.

\section{Acknowledgements}

\section{Competing interests}

The authors declare that they have no financial or personal relationship(s) that may have inappropriately influenced them in writing this article.

\section{Authors' contributions}

N.M. (University of the Witwatersrand \& Chris Hani Baragwanath Hospital) conceptualised and wrote the article and is the corresponding author. E.C. (University of the Witwatersrand) performed the literature review on PubMed and assisted in preparing the article. S.P. (Boston Children's Hospital, Harvard Medical School) provided important 
information of the ivy sign from his experience at Boston Children hospital, contributed to the images, and edited the article.

\section{References}

1. Ohta T, Tanaka H, Kuroiwa T. Diffuse leptomeningeal enhancement, 'ivy sign', in magnetic resonance images of moyamoya disease in childhood: Case report.Neurosurgery.1995;37(5):1009-1112.http.//dx.doi.org/10.1227/00006123199511000-00024

2. Maeda M, Tsuchida C. 'Ivy sign' on fluid-attenuated inversion-recovery images in childhood moyamoya disease. Am J Neuroradiol. 1999;20(10):1836-1838.

3. Yoon HK, Shin HJ, Chang YW. 'Ivy sign' in childhood moyamoya disease depiction on FLAIR and contrast-enhanced T1-weighted MR images. Radiology. 2002;223(2):384-389. http://dx.doi.org/10.1148/radiol.2232011094

4. Mori N, Mugikura S, Higano S, et al. The leptomeningeal 'ivy sign' on fluidattenuated inversion recovery MR imaging in Moyamoya disease: A sign of decreased cerebral vascular reserve? Am J Neuroradiol. 2009;30(5):930-935. http://dx.doi.org/10.3174/ajnr.A1504

5. Jin $Q$, Noguchi $T$, Irie $H$, et al. Assessment of Moyamoya disease with 3.0$T$ magnetic resonance angiography and magnetic resonance imaging versus conventional angiography. Neurol Med Chir (Tokyo). 2011;51(3):195-200. http:// dx.doi.org/10.2176/nmc.51.195

6. Kawashima $M$, Noguchi $T$, Takase $Y$, et al. Decrease in leptomeningeal ivy sign on fluid-attenuated inversion recovery images after cerebral revascularization in patients with Moyamoya disease. Am J Neuroradiol. 2010;31(9):1713-1718. http://dx.doi.org/10.3174/ajnr.A2124

7. Scott RM, Smith JL, Robertson RL, et al. Long-term outcome in children with moyamoya syndrome after cranial revascularization by pial synangiosis. Neurosurg. 2004;100(2 Suppl Pediatrics):142-149.

8. Jackson EM, Lin N, Manjila S, et al. Pial synangiosis in patients with moyamoya younger than 2 years of age. J Neurosurg Pediatr. 2014;13(4):420-425. http:// dx.doi.org/10.3171/2014.1.PEDS13251

9. Lin N, Baird L, Koss M, et al. Discovery of asymptomatic moyamoya arteriopathy in pediatric syndromic populations: Radiographic and clinical progression. Neurosurg Focus. 2011;31(6):E6. http://dx.doi.org/10.3171/2011.10.FOCUS11228 